\title{
The triggering of MHD instabilities through photospheric footpoint motions
}

\author{
C. L. Gerrard ${ }^{1}$, T. D. Arber ${ }^{2}$, and A. W. $\operatorname{Hood}^{1}$ \\ 1 School of Mathematics and Statistics, University of St Andrews, North Haugh, St Andrews, \\ Fife KY16 9SS, Scotland, UK \\ 2 Space and Astrophysics Group, Physics Department, University of Warwick, Coventry, CV4 7AL, UK
}

Received 9 July 2001 / Accepted 27 March 2002

\begin{abstract}
The results of 3D numerical simulations modelling the twisting of a coronal loop due to photospheric vortex motions are presented. The simulations are carried out using an initial purely axial field and an initial equilibrium configuration with twist, $\Phi=L B_{\theta} / r B_{z}<\Phi_{\text {crit }}$. The non-linear and resistive evolutions of the instability are followed. The magnetic field is twisted by the boundary motions into a loop which initially has boundary layers near the photospheric boundaries as has been suggested by previous work. The boundary motions increase the twist in the loop until it becomes unstable. For both cases the boundary twisting triggers the kink instability. In both cases a helical current structure wraps itself around the kinked central current. This current scales linearly with grid resolution indicating current sheet formation. For the cases studied $35-40 \%$ of the free magnetic energy is released. This is sufficient to explain the energy released in a compact loop flare.
\end{abstract}

Key words. MHD - Sun: photosphere

\section{Introduction}

The solar corona is filled with coronal loops of almost uniform cross-section. These coronal loops may be created in two ways. They may be formed by the emergence of nonpotential magnetic fields through the photosphere into the corona (Mok et al. 1997; Pevtsov et al. 1997; Longcope \& Welsch 2000; Handy \& Schrijver 2001) or they could be formed by photospheric motions twisting a potential coronal magnetic field. The footpoints of the coronal loops are embedded in the photosphere and, since the photosphere is much denser than the corona $\left(\rho=8 \times 10^{-5} \mathrm{~kg} \mathrm{~m}^{-3}\right.$ in the photosphere compared to $1 \times 10^{-11} \mathrm{~kg} \mathrm{~m}^{-3}$ in the corona), photospheric motions can twist the loop, taking it through a sequence of nearly force-free equilibria. These motions are not only a possible mechanism for forming a coronal loop, they also provide a possible mechanism for triggering MHD instabilities. The twisting of the magnetic field allows magnetic energy to build up in a loop, but once the twist $\left(\Phi=L B_{\theta} / r B_{z}\right)$ has exceeded a critical value, $\Phi_{\text {crit }}$, the loop may become unstable, releasing magnetic energy (Raadu 1972; Hood \& Priest 1979). Another possible mechanism for triggering this instability is that a loop will emerge into the corona already twisted. It will rise and as it rises the twist, $\Phi$, will increase because the

Send offprint requests to: C. L. Gerrard, e-mail: cath@mcs.st-and.ac.uk length of the loop, $L$ increases. The results reported in this paper are most applicable to the twisting of a loop due to photospheric motions. However they will be quantitatively similar to the situation where $L$ increases, since both situations involve the slow evolution of the loop beyond marginal stability.

Previous work (Galsgaard \& Nordlund 1997; Velli et al. 1997; Lionello et al. 1998; Lionello et al. 1998b; Arber et al. 1999; Baty 2000a,b; Gerrard et al. 2001) has suggested that during the non-linear evolution of such an instability a current sheet may form. However, other authors (Baty \& Heyvaerts 1996; Baty 1997; Baty et al. 1998) have found that a finite current concentration forms. At this point it may be useful to explain the difference between a current sheet and a current concentration. A current concentration is a large build up of current but the current will saturate at some finite value. For a current sheet the current density is infinite and, therefore, there is no saturation. Numerically, a current sheet can be recognized by the fact that the maximum value of the current will increase with higher grid resolution. The effect of the initial equilibrium configuration on current sheet formation is more complicated than was previously believed (Gerrard et al. 2001; Ali \& Sneyd 2001). However, it does appear that for certain configurations current sheets do form during the non-linear evolution of the kink instability. This is important since it implies that magnetic reconnection can occur 
and be driven on a timescale of the order of the Alfvén timescale. The value of the coronal resistivity is unknown but if the instability does produce a current sheet then reconnection will always occur, regardless of the value of the resistivity. Those simulations of instabilities in coronal loops which have included resistivity suggest that if current sheets do form then reconnection will occur and will release approximately half the free magnetic energy (Gerrard et al. 2001; Baty 2000a,b; Arber et al. 1999). The free magnetic energy is defined as the energy stored in the $B_{\theta}$ component of the equilibrium magnetic field. This energy is comparable to that released in a compact loop flare. A coronal loop with width $10^{6} \mathrm{~m}$, length $10^{7} \mathrm{~m}$ and magnetic field strength $100 \mathrm{G}$ (Shimizu 1996) can release $5 \times 10^{28}$ ergs and there is sufficient energy released due to the triggering of an MHD instability to explain this energy release. There are of course other possible explanations for compact loop flares but the present study does show that triggering the MHD $m=1$ instability cannot be ruled out as it operates on the correct timescales and releases the right amount of energy.

Previous work suggests that a loop whose twist has exceeded $\Phi_{\text {crit }}$ will be unstable to the $m=1$ kink instability. For some initial field configurations this instability will cause current sheets to form and reconnection will be driven in these current sheets. However, these simulations began with the loop already containing more than the critical amount of twist (Velli et al. 1997; Lionello et al. 1998; Lionello et al. 1998b; Baty \& Heyvaerts 1996; Baty 1997; Baty et al. 1998; Arber et al. 1999; Baty 2000a,b; Gerrard et al. 2001). In this paper we wish to consider the effect of twisting a loop from an initial state in which it does not contain sufficient twist to be unstable. We will then investigate how the twisting motions affect the loop and whether it does become unstable and evolve in the manner suggested by the previous simulations. It has been speculated that if the loop evolves slowly through the marginally stable point then it may not go violently unstable but simply move into a nearby helical equilibrium. This is the crucial point addressed in this paper.

Some theoretical studies of the effects of slow photospheric vortex motions on a coronal loop have been carried out. Lothian \& Hood (1989) investigated a variety of twist profiles for a toroidal loop using a Fourier-Bessel series expansion. They found that a photospheric boundary layer was present at both ends of the loop in all cases and that outside this layer the field was cylindrically symmetric. The contracted region of the loop could be described by a constant cross-sectional area. They suggested that these results for small twist would also hold for larger values of twist and that the same type of boundary layer would be present.

Steinolfson \& Tajima (1987) carried out 2D axisymmetric simulations to investigate the response of the magnetic field in a coronal loop to photospheric motions. They suggested that the motions will cause a build up of energy and that an ideal MHD instability will release the energy. However, they only considered the build up stage of the evolution. They found that the photospheric motions did increase the free magnetic energy.

Browning \& Hood (1989) used a numerical code to solve the non-linear, 2D, axisymmetric Grad-Shafranov equation and investigated the magnetostatic equilibrium of a coronal loop twisted by slow photospheric motions. They found that the core of the loop contracts while the outer part expands. Again they found that, when the length of the loop is greater than its radius, the majority of the loop can be described by a constant cross-sectional area. Browning \& Hood (1989) compared their results to $1 \mathrm{D}$ results and concluded that the agreement between the two approaches is excellent except in a small boundary layer close to the footpoints.

Robertson et al. (1992) again considered slow photospheric motions. They carried out 2D time dependent simulations and showed that 1D equilibria are a good approximation to the slow 2D evolution. Finally, they considered the $2 \mathrm{D}$ stability of coronal loops as they are twisted. As in the two papers discussed above Robertson et al. (1992) find that the loop has a constant cross-sectional area except in small boundary layers near the photosphere. They did not find any dynamic behaviour of the loop in the 2D and 1D simulations. They, therefore, concluded that any dynamic behaviour must result from a 3D instability. They then calculated the upper and lower stability bounds for a $3 \mathrm{D}$ instability.

There have been very few $3 \mathrm{D}$ numerical studies of the effect of the twisting of a loop due to photospheric motions. Mikic et al. (1990) carried out 3D MHD simulations to model the evolution of a coronal loop due to localised photospheric vortex flows. They began with a purely axial field which was then twisted by motions applied at both ends of the loop. They found the same compression of the loop as was suggested by the theoretical results and 2D simulations of Lothian \& Hood (1989), and Browning \& Hood (1989). They also found that for large aspect ratio loops the equilibrium field is independent of $z$ over most of the loop except in the boundary layer near the photosphere. Again this was in agreement with previous results. Mikic et al. (1990) also considered the linear stability of the loop formed by the twisting motions. They found that the critical twist was $\Phi_{\text {crit }}=4.8 \pi$ on the axis. Once the twist exceeded this value the loop became unstable to the kink instability. However, the self-consistent non-linear evolution of the twisted loop was not followed and so no conclusion about current sheet formation or energy release could be drawn.

Galsgaard \& Nordlund (1997) investigated the development of the internal and external kink for a straight flux tube stressed by oppositely directed rotating motions at the boundaries. They solved the resistive MHD equations in 3D and investigated the topological and energetic aspects of the non-linear evolution of the kink instability. The boundary motion was such that it introduced an internal shear in the tube as well as the overall twist. They found that, while the average Poynting flux after the instability was similar for both internal and external kink, 
there was a qualitative difference in the level of joule dissipation. They suggest that the internal kink is of most interest in relation to coronal loop heating. After the instability it quickly reaches a state of near balance between boundary work and dissipation. They conclude that these experiments suggest that the rotation or shear at the footpoints could be a source of quasi-steady heating of coronal loops.

It can be seen, therefore, that while Mikic et al. (1990) considered twisting motions they only carried out a linear investigation of the instability. The full non-linear evolution was followed by Galsgaard \& Nordlund (1997) but they concentrated on the steady-state, long term energy release and didn't give details of the earlier evolution. In this paper we have followed the full non-linear evolution and have carried out scaling experiments to study current sheet formation. We have then investigated the energy release from a single kink instability event.

Klimchuk et al. (2000) carried out 3D simulations of a loop embedded within a larger dipole configuration. Their simulations showed that twisting such a loop produces a circular cross-section. They, therefore, suggest that photospheric twisting may provide an explanation for the observed constant cross-section of coronal loops.

In this paper we neglect the toroidal curvature of the coronal loop. We investigate two cases. In case 1 we take a purely axial magnetic field and apply twisting motions at the lower and upper boundaries following Mikic et al. (1990). In case 2 we take a twisted loop for which $\Phi<\Phi_{\text {crit }}$. We then apply boundary motions to increase the twist beyond the critical value. In particular, we investigate whether applying the boundary conditions to model photospheric twisting will cause the loop to become unstable to the kink instability. We then investigate whether the instability will evolve in a similar manner to that suggested by simulations for which the initial equilibrium configuration has $\Phi>\Phi_{\text {crit }}$ or whether the slow evolution through marginal stability will cause it to evolve to a new, nearby, stable equilibrium.

\section{Numerical details}

The evolution of the coronal loop is modelled by the MHD equations,

$$
\begin{aligned}
& \frac{\partial \rho}{\partial t}=-\nabla \cdot(\rho \boldsymbol{v}), \\
& \frac{\partial}{\partial t}(\rho \boldsymbol{v})=-\nabla \cdot(\rho \boldsymbol{v} \boldsymbol{v})+\frac{1}{\mu_{0}}(\nabla \times \boldsymbol{B}) \times \boldsymbol{B}-\nabla P, \\
& \frac{\partial \boldsymbol{B}}{\partial t}=\nabla \times(\boldsymbol{v} \times \boldsymbol{B})-\nabla \times\left(\eta \frac{\nabla \times \boldsymbol{B}}{\mu_{0}}\right), \\
& \frac{\partial}{\partial t}(\rho \epsilon)=-\nabla \cdot(\rho \epsilon \boldsymbol{v})-P \nabla \cdot \boldsymbol{v}+\eta j^{2},
\end{aligned}
$$

with specific energy density,

$$
\epsilon=\frac{P}{(\gamma-1) \rho}
$$

$\boldsymbol{B}$ is the magnetic field, $\boldsymbol{j}=(\nabla \times \boldsymbol{B}) / \mu_{0}$ is the current density, $\boldsymbol{v}$ is the velocity, $P$ is the thermal pressure, $\epsilon$ is the specific energy density $(\gamma=5 / 3), \rho$ is the mass density, $\eta$ is the resistivity, and $\mu_{0}=4 \pi \times 10^{-7}$ is the magnetic permittivity. We ignore the effects of thermal conduction, radiation and heating, apart from ohmic heating. Also, since the scale height in the corona is relatively large (approximately $100 \mathrm{Mm}$ ) compared to the height of the loops $(10-50 \mathrm{Mm})$, we neglect the effect of gravity.

The equations are made dimensionless by setting, $\boldsymbol{r} \longrightarrow r^{*} \tilde{\boldsymbol{r}}, \quad \boldsymbol{B} \longrightarrow B^{*} \tilde{\boldsymbol{B}}, \quad \boldsymbol{v} \longrightarrow v_{\mathrm{A}} \tilde{\boldsymbol{v}}$, $P \longrightarrow P^{*} \tilde{P}, \quad t \longrightarrow t^{*} \tilde{t}, \quad \rho \longrightarrow \rho^{*} \tilde{\rho}, \quad \eta \longrightarrow \eta^{*} \tilde{\eta}$, where a tilde denotes a dimensionless variable. $v_{\mathrm{A}}$ is the Alfvén speed given by $v_{\mathrm{A}}=B^{*} / \sqrt{\mu_{0} \rho^{*}}, t^{*}=r^{*} / v_{\mathrm{A}}$ is the Alfvén transit time, $P^{*}=B^{* 2} / \mu_{0}$. Often the normalisation adopted in MHD studies is to take $\eta^{*}=\mu_{0} r^{*} v_{\mathrm{A}} / S$ so that for uniform resistivity we can write Eq. (3) as,

$$
\frac{\partial \boldsymbol{B}}{\partial t}=\nabla \times(\boldsymbol{v} \times \boldsymbol{B})+\frac{1}{S} \nabla^{2} \boldsymbol{B}
$$

where $S$, the Lundquist number is defined as $S=\tau_{\mathrm{d}} / \tau_{\mathrm{A}}$ where $\tau_{\mathrm{A}}$ is the Alfvén timescale and $\tau_{\mathrm{d}}=L^{2} / \eta$ is the magnetic diffusion timescale. However we do not use uniform resistivity and consequently we have kept a normalised $\eta$ explicitly in the equations by taking $\eta^{*}=$ $\mu_{0} r^{*} v_{\mathrm{A}}$. This is then chosen to prevent $|j|$ from greatly exceeding $j_{\text {grid }}$ as discussed below. Thus, we obtain the dimensionless equations, removing the tildes from the dimensionless quantities,

$\frac{\partial \rho}{\partial t}=\nabla \cdot(\rho \boldsymbol{v})$

$\frac{\partial}{\partial t}(\rho \boldsymbol{v})=-\nabla \cdot(\rho \boldsymbol{v} \boldsymbol{v})+(\nabla \times \boldsymbol{B}) \times \boldsymbol{B}-\nabla P$,

$\frac{\partial \boldsymbol{B}}{\partial t}=\nabla \times(\boldsymbol{v} \times \boldsymbol{B})-\nabla \times(\eta \nabla \times \boldsymbol{B})$,

$\frac{\partial}{\partial t}(\rho \epsilon)=-\nabla \cdot(\rho \epsilon \boldsymbol{v})-P \nabla \cdot \boldsymbol{v}+\eta j^{2}$.

In the following sections we will distinguish between an ideal MHD phase and a resistive MHD phase. In both cases these are intended as a shorthand for a more complicated description of the numerical properties of the code, how resistivity is implemented in the code and the underlying physics. In the initial phase of the simulations the loops are being slowly twisted by photospheric motion, MHD waves are present and the fields are evolving but there are no structures on short scales. Since the coronal resistivity is so low this phase would be accurately described by ideal MHD in which there was neither viscosity nor resistivity, i.e. no dissipation. The code has no explicit dissipation during this phase and will thus converge to the ideal MHD result in the limit of infinite resolution. In practice of course there is always some numerical dissipation due to the finite accuracy of the discrete representation of continuous variables. However, by repeating simulations on higher resolutions we can be sure that this numerical dissipation is not effecting the results by continuing this 
process until the higher resolution runs show no significant difference from those of a lower resolution. In this context significant features are the onset time of the instability, the growth rate and kinetic/magnetic energy at the instant the resistivity is turned on. Hence, even though there is always some numerical dissipation present during this phase we call this the ideal MHD phase as there are no unresolved structures and through convergence tests we can be sure that the numerical dissipation is unimportant.

A short time after the onset of the $m=1$ instability helical current concentrations begin to form. If these are the early manifestations of a current sheet forming then the current in these regions would grow without bound. As discussed above, this would reach a level at which resistive effects could not be ignored and the MHD instability would drive reconnection at these locations. This increase of the current density can also be viewed as a collapse of the scalelength of the magnetic field variations. Numerically, once this scalelength approaches the grid spacing we apply sufficient local resistivity to quench the current density at that value. This is implemented by estimating the maximum current allowed on the grid to be $j_{\text {grid }}$, where $j_{\text {grid }}=|B| /(\mathrm{d} x)$ and $\mathrm{d} x$ is the local grid spacing. If the local value of $|j|$ exceeds $j_{\text {grid }}$ then the resistivity is set to $\mathrm{d} x$ in that cell. This value of resistivity ensures that the CFL condition still limits the timestep to $\mathrm{d} x$, not $\mathrm{d} x^{2}$, and that any excess current density, i.e. $|j|-j_{\text {grid }}$, is dissipated in approximately one step. Hence, the current is quenched at $j_{\text {grid }}$. Note that $j_{\text {grid }}$ is itself a function of position and time since regions in which the magnetic field is large can support a larger resolved current than those with smaller magnetic field. Once the resistivity is turned on by this process we call this the resistive phase. The ideal phase had the desirable property that consistency of the numerical scheme guarantees that the result would converge to the ideal MHD result. In the resistive phase however the resistivity is grid dependent and thus the results from this phase will not converge to the exact resistive MHD result with a fixed resistivity. This does not prevent this approach from giving quantitatively accurate results for the energy release etc. during the resistive phase provided that the results do not change with grid resolution. Previous work in this area by Arber et al. (1999) showed that the resistive dissipation in current sheets driven by MHD instabilities was insensitive to the choice of resistivity. Physically this is justified if all of the free magnetic energy which is pushed by the instability into the current sheet is dissipated in that region. This would be true of anomalous resistivity, such as that generated by ion-acoustic turbulence. In such circumstances it is the rate at which flux is moved into the current sheet which determines the energy release rate. Since the rate of flow of flux into the current sheet region is determined by the large scale MHD instability this is resolved and consequently provided the resistivity is sufficient to dissipate the inflow of the magnetic field the energy release will actually be independent of that resistivity (Biskamp 1993). Only if the energy released is not constant across resolutions, i.e. converged, are runs with fixed constant resistivity necessary.

The simulations are carried out using the 3D MHD, Lagrangian remap, shock capturing code (Lare3d) described in Arber et al. (2001). The Lagrangian step is fully $3 \mathrm{D}$, uses the predictor-corrector method and artificial viscosity. The remap step uses Van Leer gradient limiters (Van Leer 1979) to ensure that it is monotonicity preserving. Furthermore, Lare3d uses Evans \& Hawley constrained transport (Evans \& Hawley 1988) to guarantee that if $\nabla . \boldsymbol{B}$ is initially zero it is maintained at zero to machine precision throughout the evolution. The numerical grid is staggered so that the density, pressure and specific energy density are defined at the cell centres; the velocities at the vertices; the magnetic field components at the cell faces and the current components along the edges of the numerical cell. $|j|=\left(j_{x}^{2}+j_{y}^{2}+j_{z}^{2}\right)^{\frac{1}{2}}$ and the resistivity are defined at the same vertices as the velocities. The staggered grid reduces the amount of averaging required in some of the calculations, thus reducing the associated error, and removes chequerboard biasing. Further details of the code are given in Arber et al. (2001).

The code is written in Cartesian co-ordinates with the numerical box stretching from $-L_{x} / 2$ to $L_{x} / 2$ and $-L_{y} / 2$ to $L_{y} / 2$. The values of $L_{x}$ and $L_{y}$ are chosen such that the boundary conditions imposed in the $x$ and $y$ directions have no effect on the evolution of the loop, which remains localised within a smaller region. Line-tying boundary conditions are applied to the magnetic field at $z=-L_{z} / 2$ and $z=L_{z} / 2$ and the velocities at the lower and upper boundaries are chosen to model photospheric motions.

\section{Initial equilibrium}

In this paper we present the results of numerical simulations carried out using two initial equilibrium configurations. In both cases photospheric vortex motions are modelled by applying a twisting velocity of the form,

$v_{\theta}=\left\{\begin{array}{cl}v_{0} \frac{r}{r_{0}}\left(1-\frac{r^{2}}{r_{0}^{2}}\right)^{2} & r<r_{0}, \\ 0.0 & r \geq r_{0},\end{array}\right.$

in opposite directions on the upper and lower boundaries, following Mikic et al. (1990). For all of the simulations $r_{0}$ is taken to be 1 (i.e. the dimensional radius is $r^{*}$ ), thus the twisting is localised within this radius. The numerical grid is stretched such that $50 \%$ of the grid points lie within $r=1$ giving higher resolution within this region. The boundaries in the $x$ and $y$ direction are positioned at a distance $\left(L_{x}=L_{y}=5\right)$ such that the boundary conditions in those directions will not affect the evolution of the loop. The magnetic field is line-tied at the upper and lower boundaries.

\subsection{Case 1}

We take an initially purely axial magnetic field,

$B_{x}=0, \quad B_{y}=0, \quad B_{z}=B_{0}=1$, 


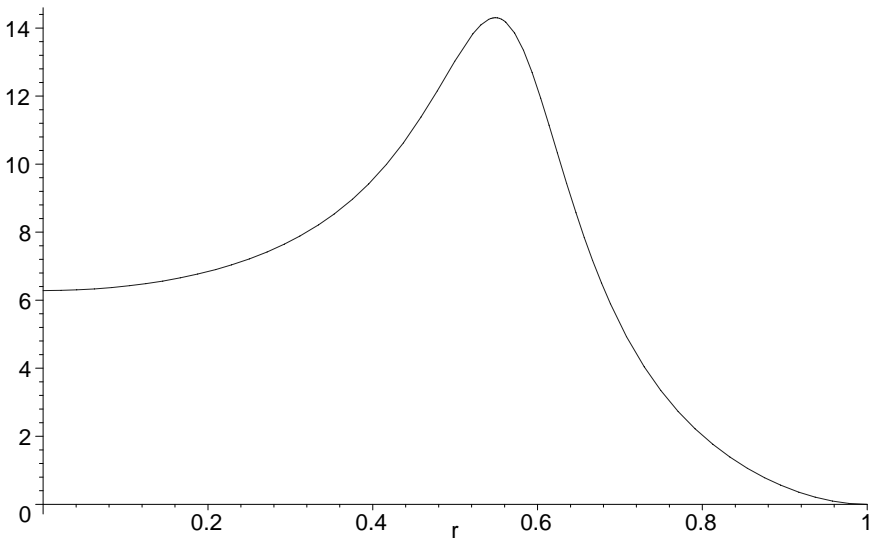

Fig. 1. A plot of the twist against radius for case 2.

with uniform density $(\rho=1)$ and pressure given by a plasma $\beta$ of $10^{-2}$. The vortex motions are applied at the boundaries such that the field is twisted in a clockwise direction on the upper boundary and in an anti-clockwise direction on the lower boundary. $L_{z}$ is taken to be $2 \pi$.

\subsection{Case 2}

Here we begin the simulations with a twisted straight cylinder given by,

$B_{\theta}=\left\{\begin{array}{cc}r\left(1-r^{2}\right)^{2} & r<1.0, \\ 0.0 & r \geq 1.0 .\end{array}\right.$

This gives $B_{\theta}=0.0$ and $j_{z}=0.0$ at $r=1.0$ - there is no net axial current in the loop. The axial field component is obtained from,

$B_{z}^{2}=B_{0}^{2}-B_{\theta}^{2}-\int_{0}^{r} 2 \frac{B_{\theta}^{2}}{u} \mathrm{~d} u$

as,

$B_{z}=\left\{\begin{array}{cc}\sqrt{B_{0}^{2}-\frac{1}{5}-r^{2}\left(1-r^{2}\right)^{4}+\frac{1}{5}\left(1-r^{2}\right)^{5}} & r<1.0, \\ \sqrt{B_{0}^{2}-\frac{1}{5}} & r \geq 1.0 .\end{array}\right.$

$B_{z}^{2}$ must remain positive hence we choose $B_{0}=0.5$, and run the simulation for a uniform density of 1.0 and pressure given by a plasma $\beta$ of $10^{-3}$. This initial equilibrium configuration gives the twist profile shown in Fig. 1. This is Equilibrium 1 from Gerrard et al. (2001).

From the linear results for this equilibrium (Van der Linden, private communication, 1999) we know that the dimensionless critical length of the loop is 4.4 . We, therefore, take a length which is shorter than this, $L_{z}=\pi$ to guarantee that the loop is initially stable.

\section{Results}

\subsection{Case 1}

\subsubsection{Ideal evolution and current sheet scaling}

In this case we begin with a purely axial field. The motions applied at the boundaries twist this field forming a
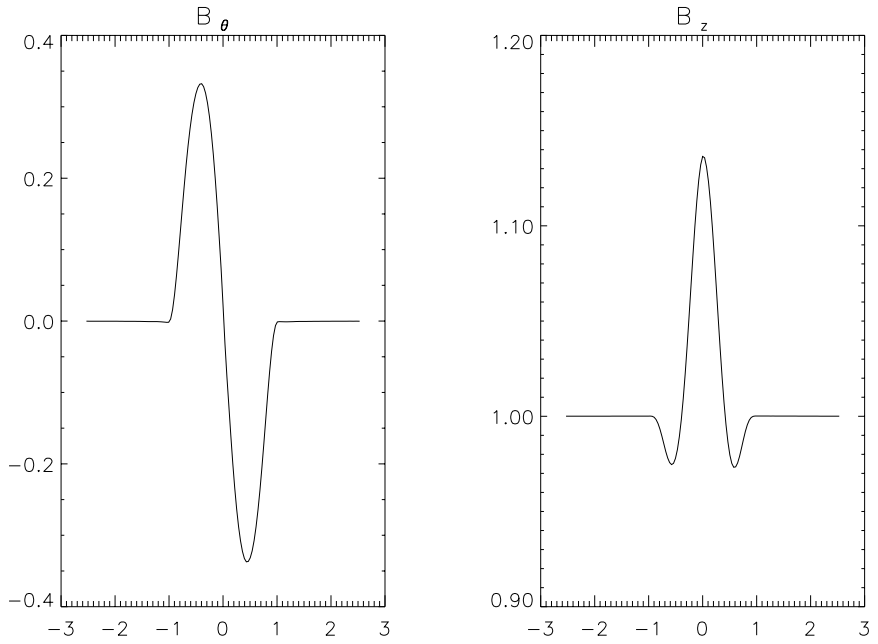

Fig. 2. $B_{\theta}$ (LHS) and $B_{Z}$ (RHS) plotted against $x$ for the initially axial loop at $t=50$ Alfvén times. These are plotted at the centre of the loop $(z=0)$ but their form does not vary with $z$.

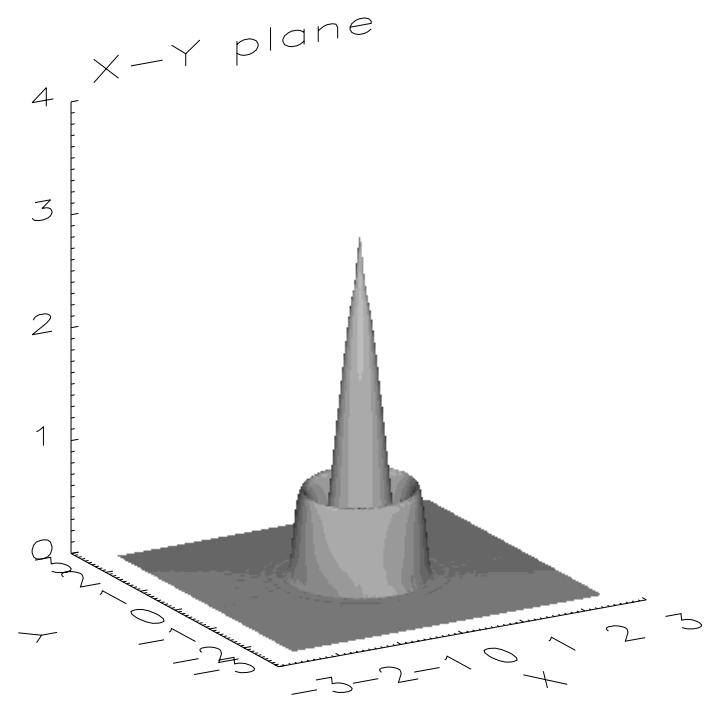

Fig. 3. A surface plot of $|j|$ at $z=0$ for the initially axial loop at $t=50$ Alfvén times.

current loop and then continue to increase the twist in the loop. We carry out these simulations for $v_{0}=0.075$. To model granular motions we would require a velocity of $0.001-0.01 v_{\mathrm{A}}$, therefore, these values are approximately a factor of 10 larger than the required velocity. However, they are significantly smaller than the Alfvén speed and to take a more physical velocity would require substantial computational time. Thus, the values used are a reasonable compromise.

We carry out the simulations on an $81^{3}, 121^{3}$ and a $161^{3}$ numerical grid. During the early part of the simulations the twisting motions begin to twist the field into a loop. This gives $B_{\theta}$ and $B_{z}$ profiles as shown in Fig. 2 and a current profile as shown in Fig. 3. These are very similar to the initially unstable profiles which have been used in previous simulations (Gerrard et al. 2001; Baty 2000; Arber et al. 1999; Lionello et al. 1998; Velli et al. 1997). 


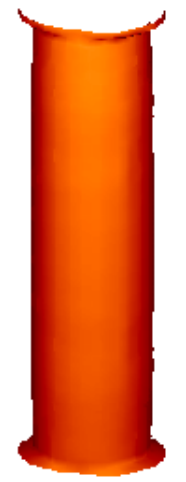

Fig. 4. An isosurface of the current during the early phase of the twisting showing the boundary layers near the photospheric footpoints.

This suggests that the previous simulations started from a reasonable initial configuration. However, these papers began with a loop which was well above the instability threshold. Our results also agree with those of Browning \& Hood (1989), Lothian \& Hood (1989) and Robertson et al. (1992) in that the loop formed by the twisting motions does have a constant cross sectional area except in a small boundary layer near the photospheric footpoints. This can be seen in Fig. 4 as an expansion near the photosphere.

The top of Fig. 5 shows the field lines at $t=0$, demonstrating that the field is purely axial. The bottom of the figure shows the field lines at $t=60$ Alfvén times. This shows that the footpoint motions are indeed twisting the field to form a current carrying loop.

Once the loop is formed we allow the twisting to continue and observe how the loop evolves. The loop can be seen to be kinked at $t=100$ Alfvén times (the lefthand side Fig. 6). This means that the loop has become unstable to the $m=1$ kink instability as suggested by previous simulations. Figure 7 shows a plot of the growth rate against time indicating that the loop has become unstable after 100 Alfvén times. The growth rate is calculated from the total kinetic energy by taking $\nabla(\log (K . E))$. The growth rate of $0.3 \tau_{\mathrm{A}}^{-1}$ is similar to the growth rate for the kink instability for the configuration investigated in Arber et al. (1999). We can compare the time for the instability to be triggered with the prediction from the linear theory. Linear theory suggests that a loop will become unstable to the kink instability when the twist exceeds $2.5 \pi$. Here, the twisting motions inject twist into the loop giving,

$\Phi=2 \int_{0}^{t} \frac{v_{\theta}}{r} \mathrm{~d} t$

SO,

$\Phi=2 v_{0} t\left(1-r^{2}\right)^{2}$.

We then calculate the approximate time at which the kink instability will be triggered. To obtain a first estimate, we integrate the twist over the cross-sectional area of the
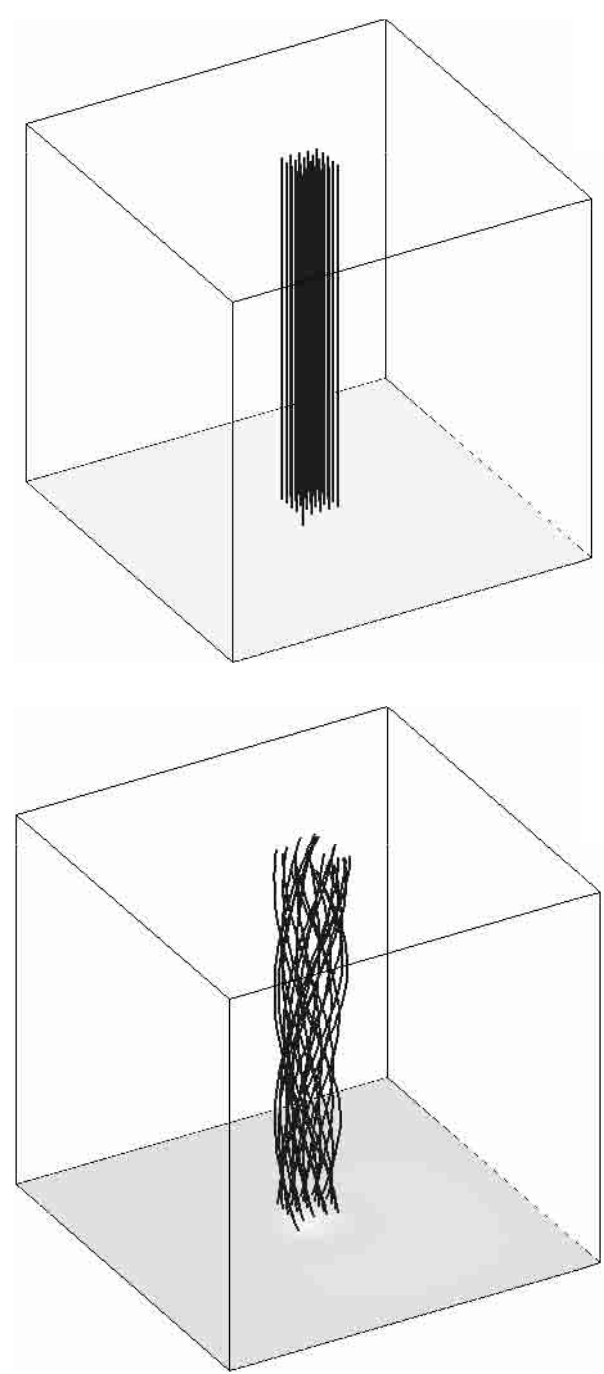

Fig. 5. A selection of fieldlines at $t=0$ (top) and at $t=60 \tau_{\mathrm{A}}$ (bottom).

loop and find the time at which the average twist is equal to $2.5 \pi$,

$\frac{\int_{0}^{2 \pi} \int_{0}^{r=1} \Phi r \mathrm{~d} r \mathrm{~d} \theta}{\pi r^{2}}=4 t v_{0} \int_{0}^{r=1}\left(1-r^{2}\right)^{2} r \mathrm{~d} r=2.5 \pi$,

so,

$t=\frac{15 \pi}{4 v_{0}}$

Here, we have $v_{0}=0.075$ which gives $t=157.1$. To calculate a second estimate, that is usually a lower limit, we calculate the time at which the maximum twist is equal to $2.5 \pi$,

$2 v_{0}\left(1-(0)^{2}\right)^{2} t=2.5 \pi$,

and obtain $t=52.3$. We can, therefore, predict that the loop will become unstable to the kink instability between $t=52.3$ and $t=157.1$ Alfvén times. The results from the simulations, which suggest that the loop becomes unstable at approximately $t=100$ Alfvén times, agree with this prediction. However, it should be noted that these 

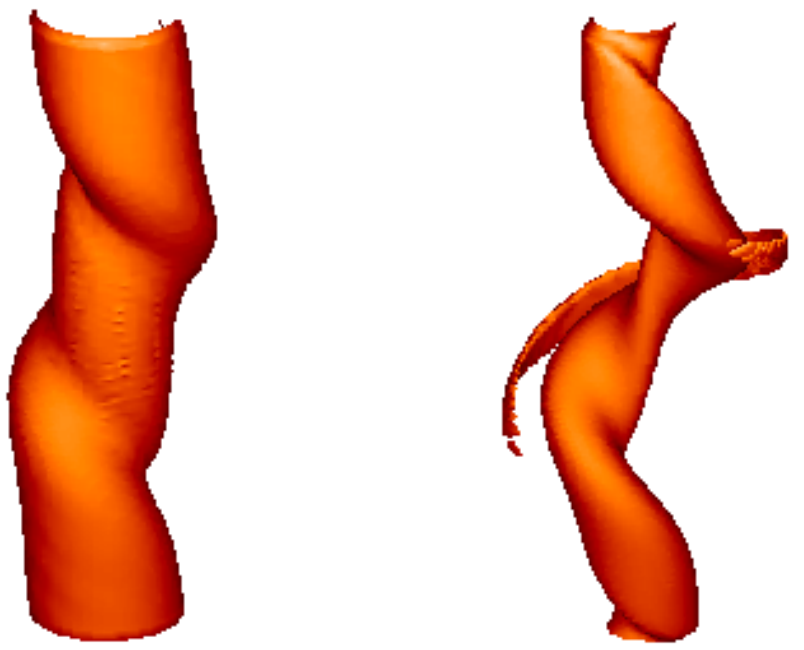

Fig. 6. An isosurface of the kinked current at $t=100$ Alfvén times (LHS) and an isosurface of the current at $t=120$ Alfvén times (RHS) showing a helical current concentration starting to form.

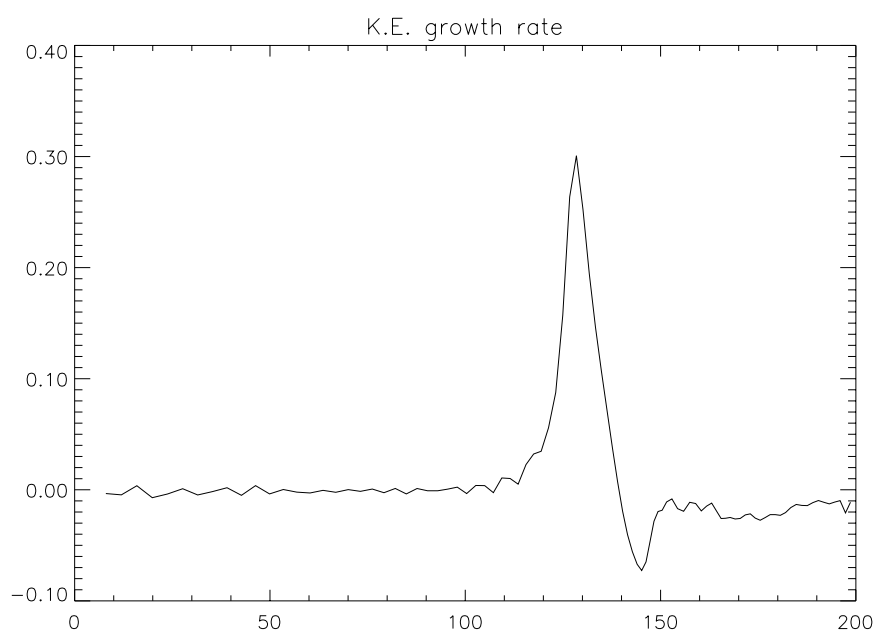

Fig. 7. Plot of the growth rate, $\gamma$, against time for the simulations for case 1.

linear predictions are very crude estimates, especially for the lower limit.

At $t=100$ Alfvén times the loop can be seen to be kinked. At later times a helical concentration of current builds up around the kinked central column (Fig. 6, RHS). To test whether this structure is a current sheet or whether the current saturates at a finite value we carry out the simulations on three different resolutions, $81^{3}, 121^{3}$ and $161^{3}$. If in all of these runs the maximum current density saturates at $j_{\text {grid }}$ then we conclude that this is consistent with the formation of a current sheet as the current has reached the maximum value allowed on that resolution. This behaviour is shown in Fig. 8 which is a plot of the current for the $81^{3}$ simulations from $t=110$ to $t=130$. The $m=1$ instability leads to a rapid rise in the maximum current density after $t=120$. At $t=122$ the resistivity is turned on to prevent increase in the current density beyond $j_{\text {grid }}$. As a final confirmation that the maximum

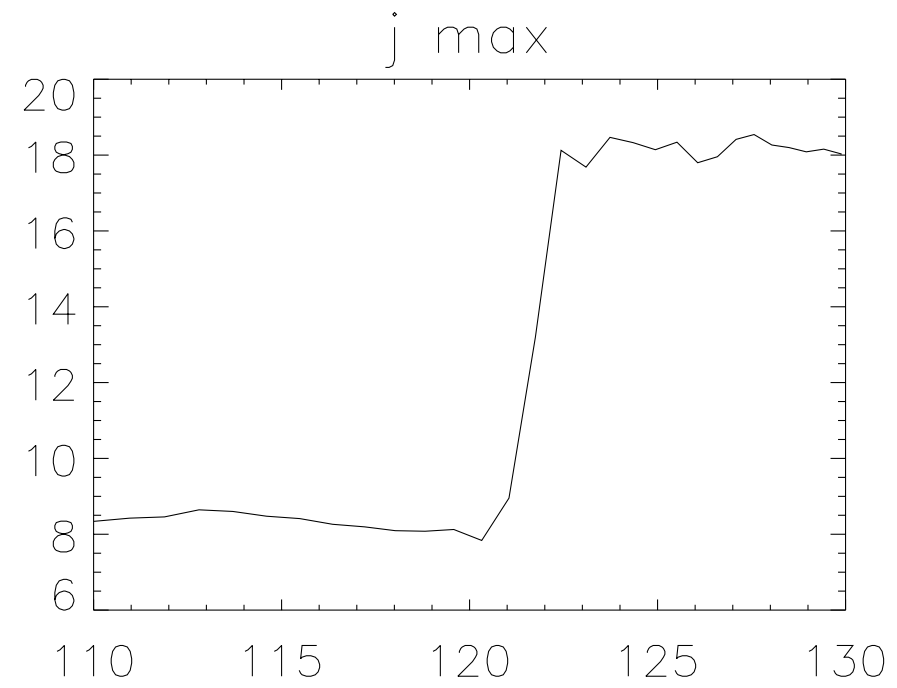

Fig. 8. Plot of maximum current against time for case 1.

Table 1. Scaling of the maximum of the current with higher resolution.

\begin{tabular}{|c|c|c|c|}
\hline \hline & \multicolumn{3}{|c|}{ grid scalings } \\
\hline \hline$n x, n y, n z$ & $81^{3}$ & $121^{3}$ & $161^{3}$ \\
$\mathrm{~d} x$ & 0.0532497 & 0.0359548 & 0.0269261 \\
\hline$j_{\max }$ & 18 & 27 & 36 \\
Expected scaling & 18.1 & 27.0 & 35.9 \\
\hline
\end{tabular}

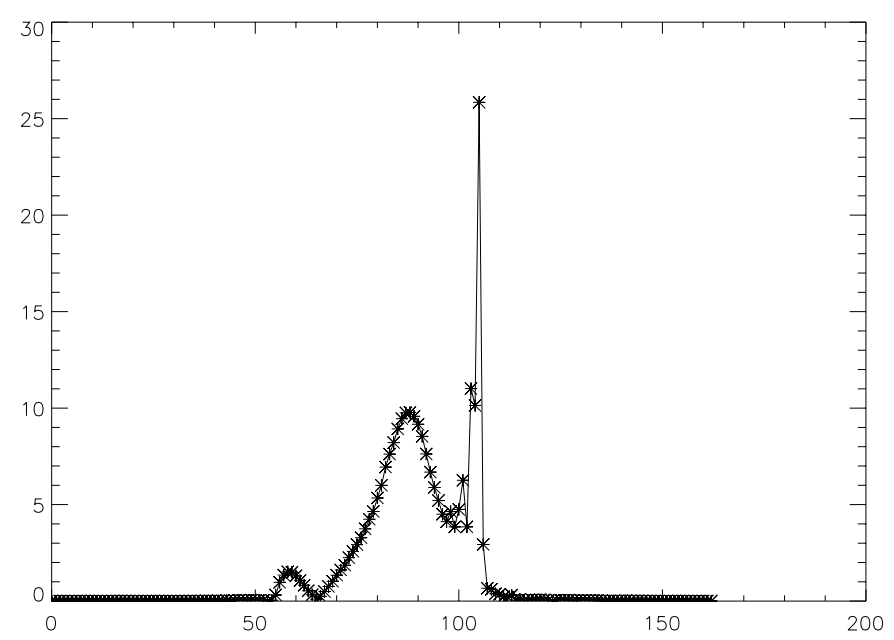

Fig. 9. A plot through the current sheet along the line $x=y$ at $z=0$ at $t=125$.

current density is scaling as a current sheet Table 1 gives the values of $\mathrm{d} x$ and $j_{\max }$ for each grid resolution. The expected scalings are based on the $121^{3}$ result and $j_{\max }$ is the maximum current density when the resistivity is turned on. As can be seen from Table 1, the current scales with higher resolution and always triggers the resistive phase, i.e. locally $|j|$ exceeds $j_{\text {grid }}$. This indicates that the structure seen in Fig. 6 is indistinguishable from a current sheet on the resolutions tested. Figure 9 is a plot through the current sheet with the grid points marked. It shows that the current sheet is only a few grid points across. 

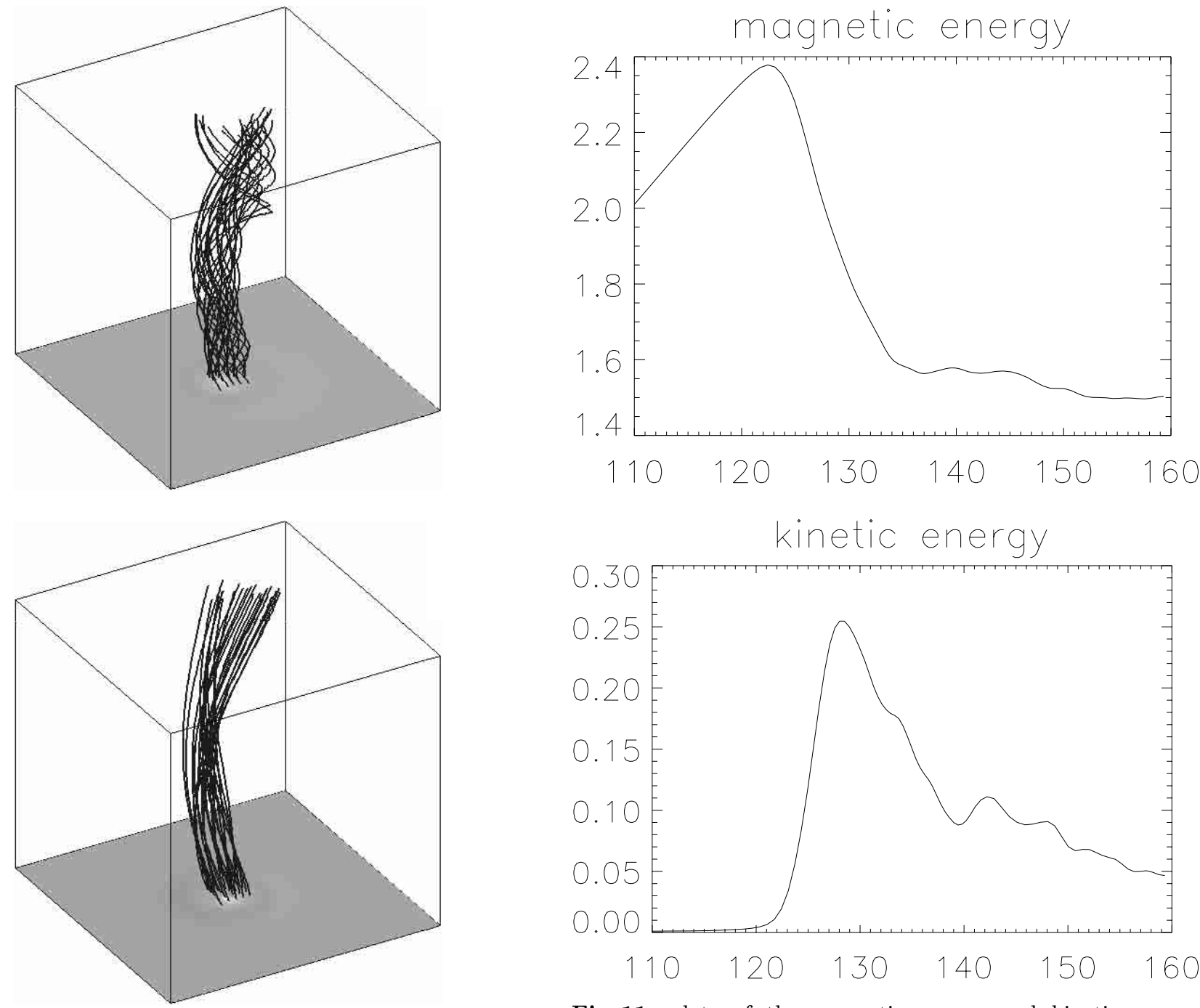

Fig. 10. A selection of fieldlines at $t=120$ (top) and at $t=$ $160 \tau_{\mathrm{A}}$ (bottom).

\subsubsection{Resistive evolution}

Since the results in Table 1 indicate formation of a current sheet we can continue the simulations into the resistive phase of the evolution and obtain quantitatively correct results. The value of the coronal resistivity is not known but is believed to be very small, smaller than the value we can use in these simulations. However in a current sheet reconnection will occur regardless of the value of the resistivity.

Here, we continue the simulations on the $81^{3}$ and $161^{3}$ grids (i.e. a doubling of grid resolution). This will allow us to check whether the value of the energy release which we obtain is the converged value.

The simulations are continued until $t=160 \tau_{\mathrm{A}}$. The free magnetic energy is taken as the magnetic energy stored in the $B_{\theta}$ component of the magnetic field. It peaks between $t=122$ and $t=123$ Alfvén times and then falls throughout the rest of the simulation (Fig. 11). The kinetic energy (Fig. 11) peaks suddenly and then falls off

Fig. 11. plots of the magnetic energy and kinetic energy against time during the resistive phase for Case 1 .

steadily. The ohmic heating increases at first rapidly and then steadies off to a constant value as shown in Fig. 12.

Figure 10 shows a selection of fieldlines at $t=120$ and at $t=160 \tau_{\mathrm{A}}$. It can be seen that the fieldlines are significantly untwisted at $t=160 \tau_{\mathrm{A}}$. This implies that reconnection has occurred. The results from the $81^{3}$ and $161^{3}$ grid suggest that approximately $38 \%$ (with a difference of $3 \%$ in the results from the grid resolutions) of the free magnetic energy was released by the reconnection.

\subsection{Case 2}

\subsubsection{Ideal evolution and current sheet scaling}

We now wish to consider the effect of photospheric motions on a loop which already contains some twist but for which $\Phi<\Phi_{\text {crit }}$. To do this we use an equilibrium which we studied in a previous paper (Gerrard et al. 2001) but take the loop to be shorter than the critical length. The boundary motions are then applied using Eq. (12) with $v_{0}=0.05$ in a clockwise direction on the lower boundary 


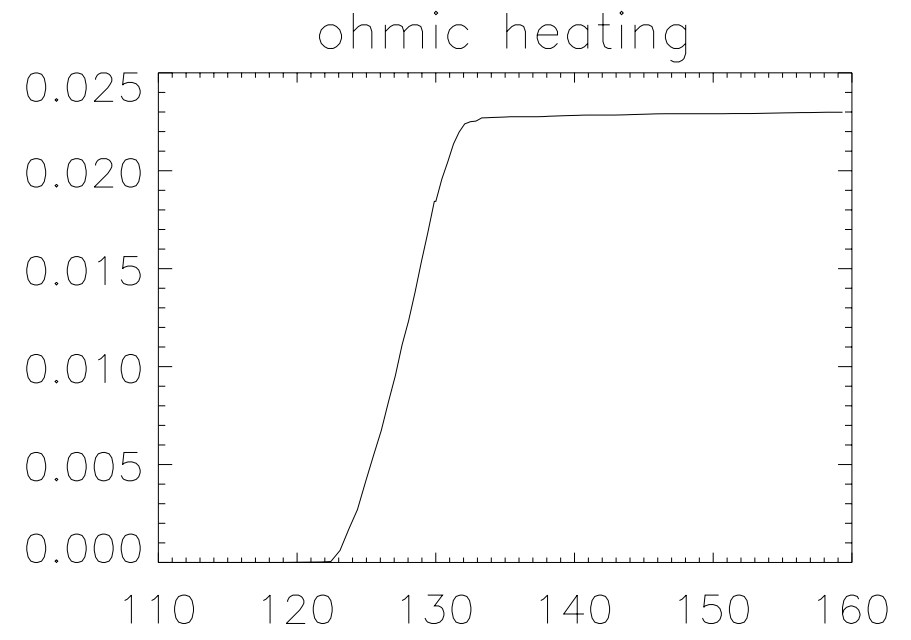

Fig. 12. A plot of the ohmic heating against time for Case 1.

and anti-clockwise on the upper boundary. These increase the twist in the loop (Fig. 13).

During the initial phase a boundary layer is again visible near the photospheric boundaries as shown in Figs. 14 and 15 . However, the boundary layer seems to have a more limited extent than for case 1 due to the fact that we have started from a twisted field.

We carry out the simulations on $81^{3}, 121^{3}, 161^{3}$ and $201^{3}$ grids. From these simulations we can see that at $t=40 \tau_{\mathrm{A}}$ the loop has become kinked, indicating that the loop has become unstable to the kink instability. Plotting the growth rate against time (Fig. 16) also shows that the loop becomes unstable to the kink instability after approximately 40 Alfvén times. Again, we compare this to the linear prediction. Here, we apply the same twisting motions as for case 1 but the loop already contains some twist. We calculate the same estimates for the time taken for the loop to become unstable as for case 1. Thus, we have,

$4 t v_{0} \int_{0}^{r=1}\left(1-r^{2}\right)^{2} r \mathrm{~d} r+2 \int_{0}^{r=1} \frac{\pi B_{\theta}}{r B_{z}} r \mathrm{~d} r=2.5 \pi$.

Evaluating this with $v_{0}=0.05$ gives $t=68.4$. For the second estimate we have a twist of,

$\Phi=2 t v_{0}\left(1-r^{2}\right)^{2}+\frac{\pi B_{\theta}}{r B_{z}}$.

This is more complicated than case 1 owing to the $B_{\theta} / r B_{z}$ contribution. However, from Fig. 1 it can be seen that the maximum of the twist in fact exceeds $2.5 \pi$ at $t=0$. We know (Van der Linden, private communication, 2000) that this configuration is stable for a loop of length less than 4.4. As mentioned for case 1 our method of approximating the stability limits is very crude and here this estimate is not very useful. Thus the linear results suggest that the loop will become unstable by $t=68.4$. As we have seen the simulations show the loop becoming unstable at $t=40$ which is in agreement with this. It is also worth noting that this linear prediction is only approximate as the loop does not have uniform twist and is not stationary.
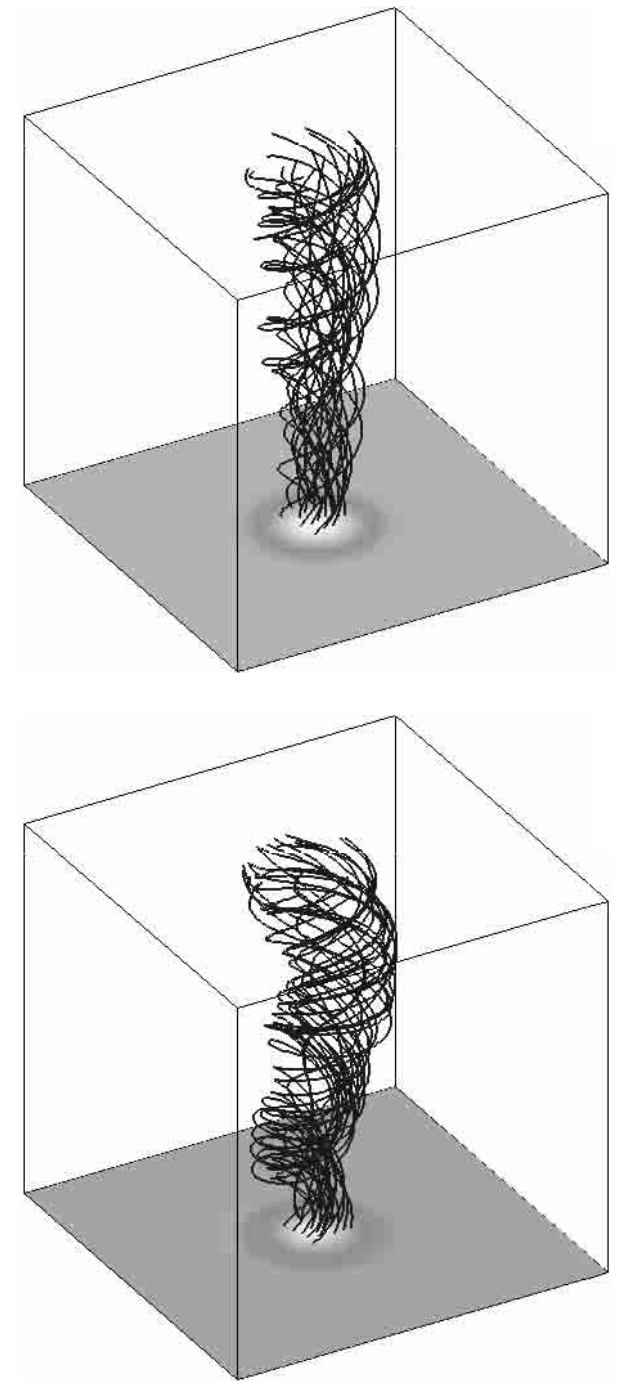

Fig. 13. A selection of fieldlines at $t=0$ (top) and at $t=50 \tau_{\mathrm{A}}$ (bottom).

Table 2. Scaling of the maximum of the current with higher resolution.

\begin{tabular}{|c|c|c|c|c|}
\hline \hline & \multicolumn{4}{|c|}{ grid scalings } \\
\hline \hline$n x, n y, n z$ & $81^{3}$ & $121^{3}$ & $161^{3}$ & $201^{3}$ \\
$\mathrm{~d} x$ & 0.0532497 & 0.0359548 & 0.0269261 & 0.0215211 \\
\hline$j_{\max }$ & 4.0 & 6.2 & 8.1 & 10.7 \\
Expected scaling & 4.1 & 6.2 & 8.2 & 10.3 \\
\hline
\end{tabular}

An isosurface of the current at $t=60 \tau_{\mathrm{A}}$ (Fig. 17) shows a helical structure wrapped around the kinked central column. As in Sect. 4.1.1 we wish to investigate whether this helical structure is a current sheet or whether the current saturates at a finite value. Table 2 gives $j_{\max }$ against $\mathrm{d} x$ for each grid resolution investigated. $j_{\max }$ is calculated as described in Sect. 4.1.1 and again $\mathrm{d} x$ is the grid spacing at the location of the current concentration formation. The predicted scalings are, as in Sect. 4.1.1, based on the $121^{3}$ results. This table shows that the maximum current is scaling linearly with grid resolution. 


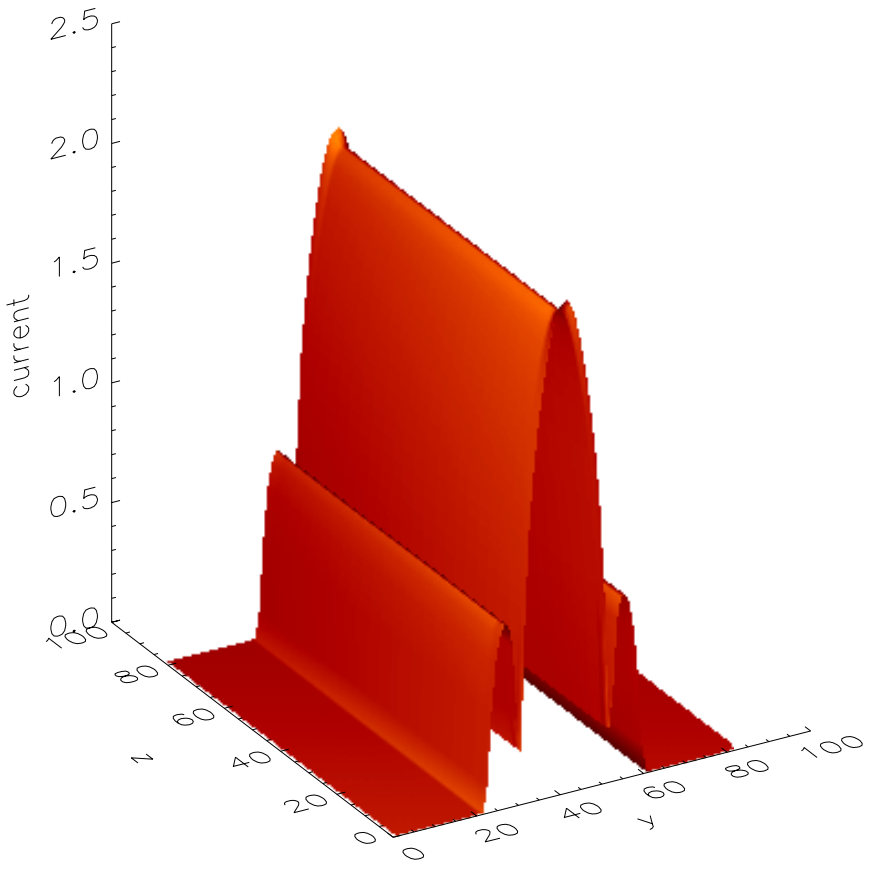

Fig. 14. A surface plot showing the boundary layers at the photospheric boundaries.

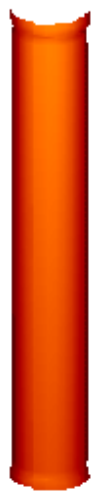

Fig. 15. An isosurface showing the boundary layers.

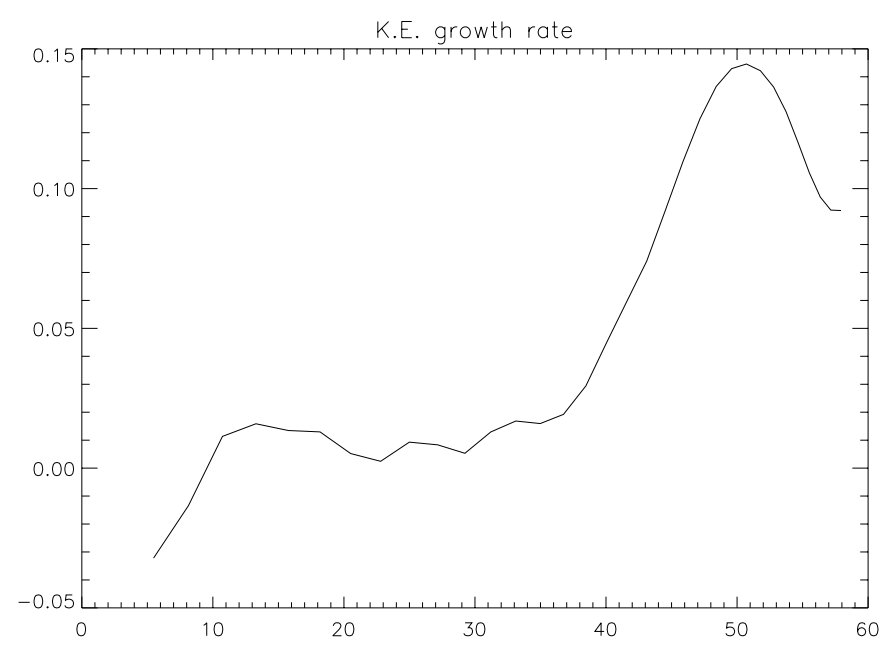

Fig. 16. Plot of the growth rate, $\gamma$, against time for the ideal simulations for case 2.

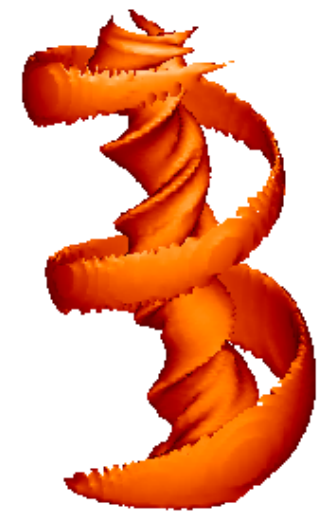

Fig. 17. An isosurface of the current for Case 2 at $t=60$ showing the kinked central column of current with a helical current concentration wrapped around it.

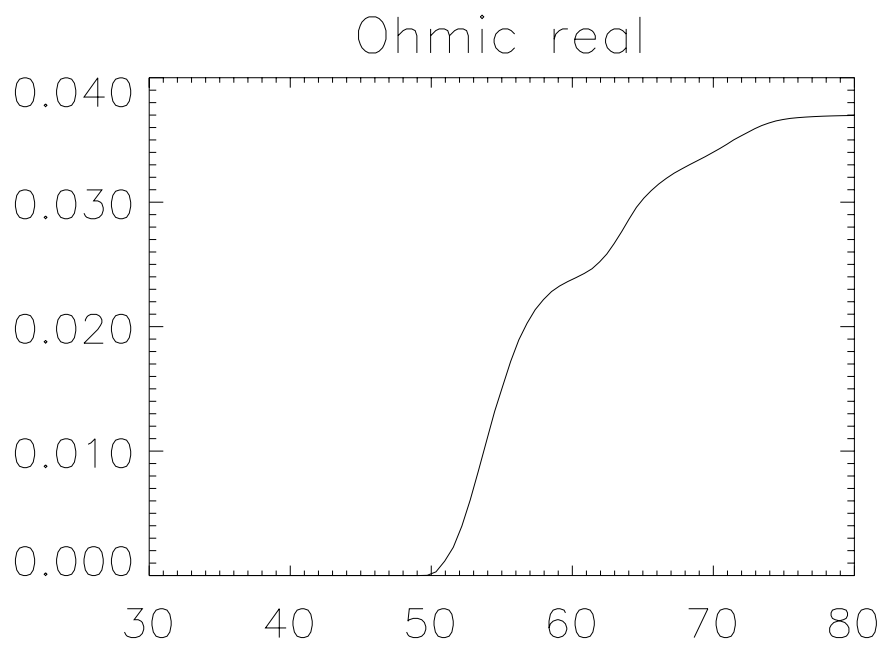

Fig. 18. A plot of the ohmic heating for Case 2 .

This is consistent with the current density reaching $j_{\text {grid }}$ and therefore indicative of a current sheet.

\subsubsection{Resistive evolution}

We now continue the simulation until the ohmic heating steadies off to a constant value, as shown in Fig. 18. This allows us to investigate the resistive phase of the evolution. As noted in Sect. 4.1.2 the value of the resistivity in the solar corona is unknown but is likely to be smaller than the value used in these simulation. However, since the results in Table 2 are indicative of current sheet formation the resistive phase simulations will be quantitatively accurate regardless of the value of the resistivity. We carry out the continuation of the simulations on $81^{3}, 121^{3}$ and $161^{3}$ grids, running the code until $t=100 \tau_{\mathrm{A}}$. This will allow us to check that the energy release is constant across grid resolutions i.e. converged, as discussed in Sect. 2.

During this resistive phase of the simulation the ohmic heating increases, at first quickly and then steadies off to a constant value, the magnetic energy decreases and the kinetic energy increases suddenly and then decreases slowly (Fig. 19). Figure 20 shows a selection of fieldlines at $t=50$ 

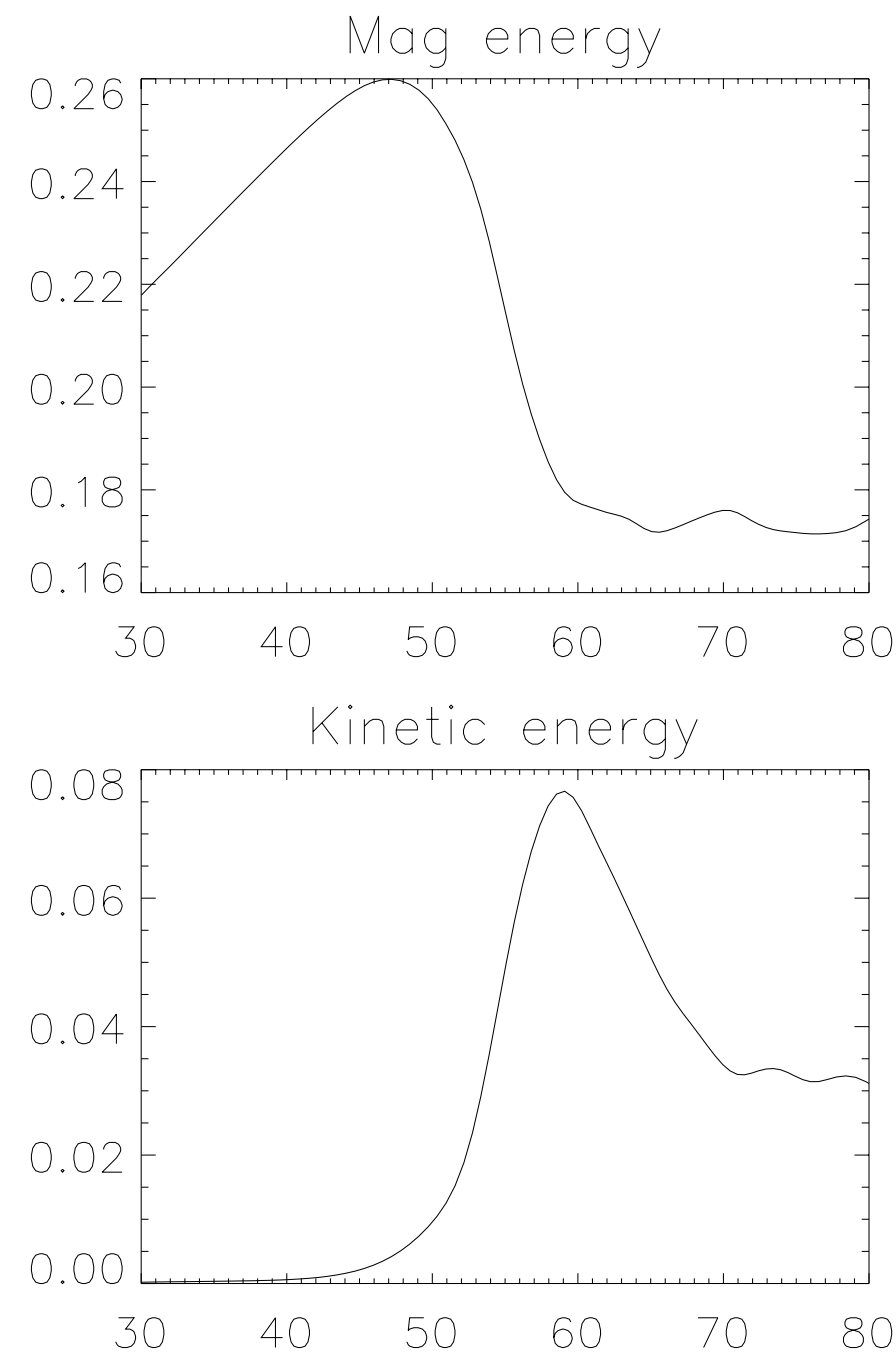

Fig. 19. plots of the magnetic energy and kinetic energy against time during the resistive phase for Case 2 .

and $t=100$ Alfvén times. It can be seen that the fieldlines have become untwisted by $t=100 \tau_{\mathrm{A}}$. This, with the decreasing magnetic energy, the sudden increase in kinetic energy and increasing ohmic heating, suggests that the fieldlines have reconnected between $t=50$ and $t=100 \tau_{\mathrm{A}}$ releasing the free magnetic energy. The free magnetic energy is calculated by taking the amount of energy stored in the $B_{\theta}$ component of the magnetic field. This peaks at approximately 48 Alfvén times and then decreases by approximately $35 \%$ (on the $81^{3}, 121^{3}$, and $161^{3}$ grids). Since all the grid resolutions result in the same energy release this is the converged value. This suggests that $35 \%$ of the free magnetic energy is released by reconnection.

\section{Conclusions}

Our aim in this paper has been to carry out non-linear 3D MHD simulations of the effect of photospheric vortex motions on the evolution of a coronal loop. In previous simulations (Gerrard et al. 2001; Baty 2000; Arber et al. 1999; Lionello et al. 1998) the initial configuration has been an equilibrium with $\Phi>\Phi_{\text {crit }}$. This unstable equilibrium is
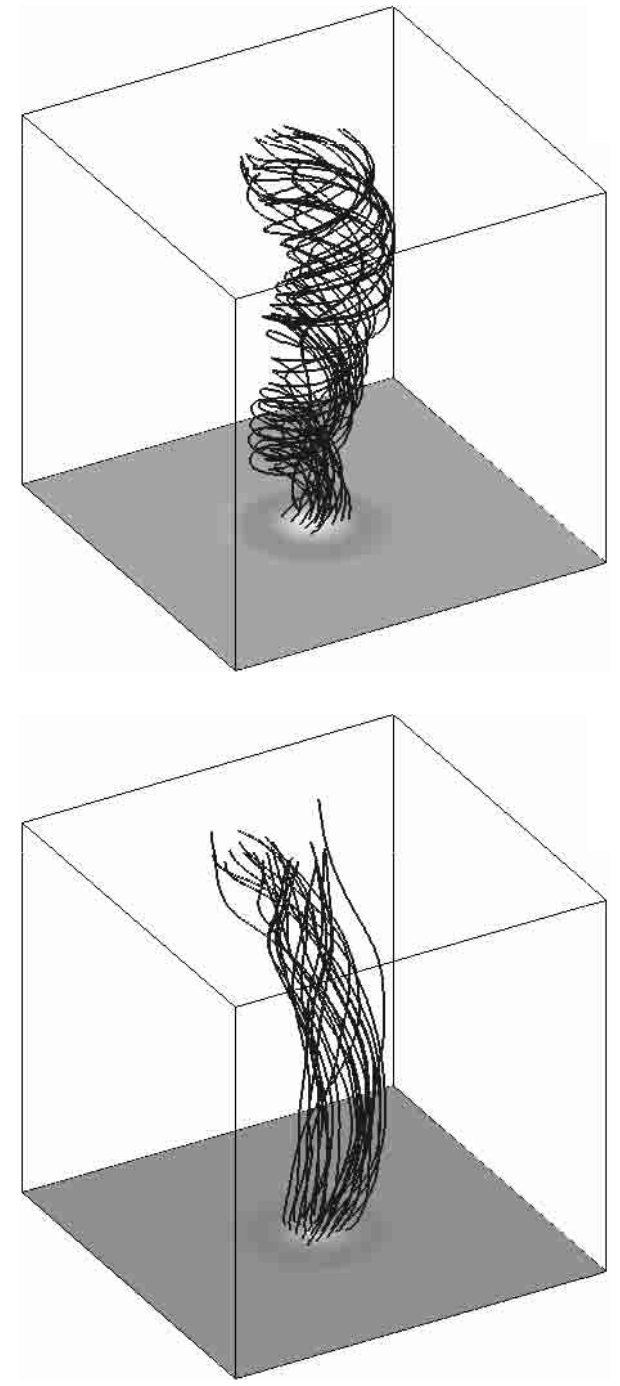

Fig. 20. A selection of fieldlines at $t=50$ (top) and at $t=$ $100 \tau_{\mathrm{A}}$ (bottom).

then perturbed, the instability grows and its evolution is followed. Figure 21 shows a sketch of the growth rate, $\gamma$ against the length $L_{z}$ of a loop (this is similar to Fig. 3 of Lionello et al. 1998). The simulations with $\Phi>\Phi_{\text {crit }}$ in the initial configuration, are represented on this plot by a cross. In this paper our aim has been to consider an earlier stage in the evolution (the region circled in the plot). By twisting a loop with velocities which mimic photospheric footpoint motions we can evolve it slowly through this early phase. This slower evolution may result in

- the formation of a stable kinked equilibrium

- the saturation of the current concentration.

The aim of this paper is to investigate what effect the boundary motions have on the evolution of the loop. In particular, we have investigated whether the twisting motions at the loop's footpoints can trigger the kink instability and whether the instability will evolve as predicted by the simulations where $\Phi>\Phi_{\text {crit }}$. i.e. whether a current sheet will form and reconnection will take place. 


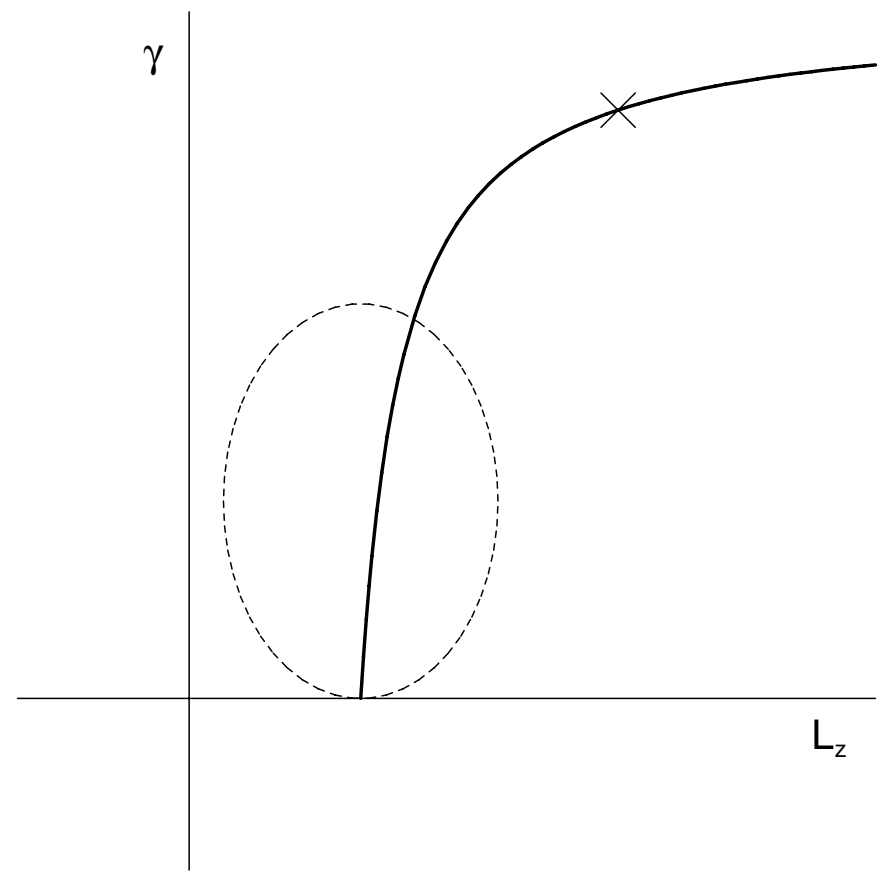

Fig. 21. Plot of $\gamma$ against $L_{z}$ with the cross indicating simulations beginning with $\Phi>\Phi_{\text {crit }}$ and the encircled region representing the simulations carried out in this paper where the loop is evolved slowly until the instability is triggered.

To do this we take two initial equilibrium configurations. In case 1 we consider a purely axial magnetic field and apply boundary conditions to model photospheric motions. These twist the field into a loop and then increase the twist in the loop until it exceeds the critical value for the kink instability. In case 2 we consider an equilibrium which already contains some twist but for which $L_{z}$ is chosen such that $\Phi<\Phi_{\text {crit }}$. The twisting motions on the boundaries are then chosen to increase the twist in the loop until it exceeds $\Phi_{\text {crit }}$. An isosurface of the current at this time shows that it has become kinked. For both case 1 and case 2 we allow the loop to continue to evolve and a helical structure is seen to wrap itself around the kinked central current. We have investigated the scaling of the current with grid resolution and have found that $j_{\max }$ scales linearly with higher resolution. Actually the current density is limited by localised resistivity to $j_{\text {grid }}$, the maximum resolvable current density on the grid. Thus, the linear scaling is simply another way of stating that the current density always reaches the maximum value allowed on each grid. This indicates that the helical structure, in both cases investigated in this paper, behaves as a current sheet on all resolutions tested. This means that we can carry out simulations with a larger value of resistivity than is likely in the solar corona but will obtain quantitatively correct results. Provided a current sheet forms in ideal MHD the results from resistive MHD simulations are not sensitive to the local value of resistivity. It is however important that the large scale structure of the ideal MHD mode is resolved as it is the rate at which this drives new flux into the resistive layer which determines the energy release rate. It should also be noted that this is best achieved by having a localised resistivity as a uniform resistivity would effect the development of the large scale structure as was observed in Arber et al. (1999).

For case 1 the resistive phase of the evolution is investigated on both an $81^{3}$ and a $161^{3}$ grid giving a doubling of grid resolution. For case 2 the simulations are continued on the $81^{3}, 121^{3}$ and $161^{3}$ grids. For both cases the simulations are continued until the ohmic heating has steadied off to a constant value. Plots of the fieldlines at the start and end of the resistive phase of the simulation show that the fieldlines are significantly untwisted by the end of the simulation. This suggests that reconnection has occurred. Free magnetic energy is released as a result of this reconnection. For both cases the energy release is the same on all three grid resolutions and therefore the value is the converged value. Thus reconnection releases $38 \%$ of the free magnetic energy for case 1 and $35 \%$ of the free magnetic energy for case 2.

In conclusion,

- During the initial twisting of case 1 and case 2 we obtain small boundary layers near the photosphere. This agrees with the results of Lothian \& Hood (1989), Browning \& Hood (1989), Robertson et al. (1992), and Mikic et al. (1990).

- For case 1 the boundary motions twist the initially axial field into a loop with a profile similar to those studied in previous simulations.

- For case 1 the boundary velocities then continue to twist the loop eventually triggering the kink instability. For both case 1 and case 2 a current concentration builds up during the non-linear evolution of the instability.

- $j_{\max }$ scales linearly with grid resolution for case 1 and case 2 indicating current sheet formation.

- For case 1 and case 2 reconnection releases, respectively, $38 \%$ and $35 \%$ of the free magnetic energy.

- This is sufficient energy to explain a compact loop flare.

Acknowledgements. The authors would like to thank the anonymous referee for their invaluable advice and help in greatly improving this paper. The simulations were carried out on the UK MHD consortium Compaq cluster at the University of St Andrews funded by JREI/SHEFC. The authors would like to thank C. Mellor for his assistance with Maple.

\section{References}

Ali, F., \& Sneyd, A. D. 2001, Geophysical and Astrophysical Fluid Dynamics, 94, 121

Arber, T. D., Longbottom, A. W., Gerrard, C. L., \& Milne, A. M. 2001, J. Comput Phys., 170, 1

Arber, T. D., Longbottom, A. W., \& Van der Linden, R. A. M. 1999, ApJ, 517, 990

Baty, H., \& Heyvaerts, J. 1996, A\&A, 308, 935

Baty, H. 1997, A\&A, 318, 621

Baty, H., Einaudi, G., Lionello, R., \& Velli, M. 1998, A\&A, 333,313 
Baty, H. 2000a, A\&A, 353, 1074

Baty, H. 2000b, A\&A, 360, 345

Biskamp 1993, Nonlinear Magnetohydrodynamics, CUP

Browning, P. K., \& Hood, A. W. 1989, Sol. phys., 124, 271

Evans, C. R., \& Hawley, J. F. 1988, AJ, 332, 659

Galsgaard, K., \& Nordlund, A. 1997, JGR, 102, 219

Gerrard, C. L., Arber, T. D., Hood, A. W., \& Van der Linden, R. A. M. 2001, A\&A, 373, 1089

Handy, B. N., \& Schrijver, C. J. 2001, ApJ, 547, 1100

Hood, A. W., \& Priest, E. R. 1979, Sol. Phys., 64, 303

Klimchuk, J. A., Antiochos, S. K., \& Norton, D. 2000, ApJ, 542,504

Lionello, R., Velli, M., Einaudi, G., \& Mikic, Z. 1998, AJ, 494, 840

Lionello, R., Schnack, D. D., Einaudi, G., \& Velli, M. 1998b, Phys. Plasmas, 5, 3722
Longcope, D. W., \& Welsch, B. T. 2000, ApJ, 545, 1089

Lothian, R. M., \& Hood, A. W. 1989, Sol. Phys., 122, 227 Mikic, Z., Schnack, D., \& Van Hoven, G. 1990, ApJ, 361, 690 Mok, Y., Van Hoven, G., \& Mikic, Z. 1997, ApJ, 490, L107 Pevtsov, A. A., Canfield, R. C., \& McClymont, A. N. 1997, ApJ, 481, 973

Raadu, M. A. 1972, Sol. Phys., 22, 425

Robertson, J. A., Hood, A. W., \& Lothian, R. M. 1992, Sol. Phys., 137, 273

Shimizu, T. 1996, in Magnetic Reconnection in the Solar Atmosphere, ed. R. Bentley, \& J. T. Mariska (San Fransisco: ASP), ASP Conf. Ser., 111, 59

Steinolfson, R. S., \& Tajima, T. 1987, AJ, 322, 503

Van Leer, B. 1979, J. Comput Phys., 32, 101

Velli, M., Lionello, R., \& Einaudi, G. 1997, Sol. Phys., 172, 257 